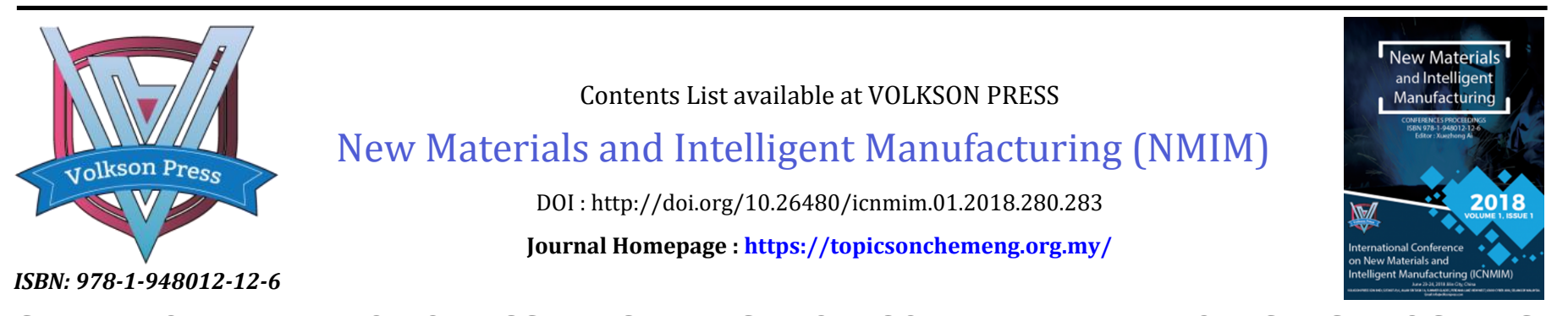

\title{
STUDY ON METABONOMICS MECHANISM OF COMPATIBILITY OF GINSENOSIDES AND TENUIGENINS ON ALZHEIMER'S DISEASE RATS BASED ON UPLC-MS
}

\author{
Yan Zhang ${ }^{*}$, Hui Li ${ }^{2}$, Guan Wang ${ }^{1}$, Ben Liu ${ }^{1}$, Fan Zhang ${ }^{1}$, Hongqi Wang ${ }^{1}$, Wunjing You ${ }^{1}$, Cong $\mathrm{Ni}^{1}$, Wenhao $\mathrm{Ma}^{1}$ \\ ${ }^{1}$ School of Chemical and Pharmaceutical Engineering, ilin Institute of Chemical Technology, No.45 Chengde Street, Jilin, China \\ ${ }^{2}$ Graduate School, Jilin Institute of Chemical Technology, No.45 Chengde Street, Jilin, China \\ *Corresponding Author Email: zyzxorange@126.com
}

This is an open access article distributed under the Creative Commons Attribution License, which permits unrestricted use, distribution, and reproduction in any medium, provided the original work is properly cited.

\section{ARTICLE DETAILS}

\section{Article History:}

Received 26 June 2018 Accepted 2 July 2018 Available online 1 August 2018

\section{ABSTRACT}

Ginseng and polygala tenuifolia are traditional Chinese medcine for the treatment of Alzheimer's disease (AD). Ginsenoside and tenuigenin are the major effective compounds found in ginseng and polygala tenuifolia, respectively. In this study, a urinary metabonomics method based on ultra-performance liquid chromatography coupled with quadrupole time-of-flight high definition mass spectrometry was performed to assess the holistic mechanism of the compatibility of ginsenosides and tenuigenins for treating AD in rats. 30 male rats were randomly divided into 3 groups, named as the normal group (NG), the model group (MG), the treatment group (TG). The MG and TG were induced for modeling $\mathrm{AD}$ by using D-galactose combined with aluminum trichloride. Multivariate statistical approaches, such as principal component analysis (PCA) and orthogonal projection to latent structures squares-discriminant analysis (OPLS-DA) were used to distinguish 3 groups and find potential biomarkers. As results shown, there was a clear separation between the 3 groups in the PCA. Ten potential biomarkers had been identified using OPLS-DA and S-plot, which belonged to tryptophan metabolism, taurine and hypotaurine metabolism and inositol phosphate metabolism. This study shows that metabonomics is a powerful methodology, which could further explore the mechanism of compatibility of ginsenosides and tenuigenins in the treatment of AD.

\section{KEYWORDS}

Compatibility of ginsenosides and tenuigenins, Alzheimer's disease, metabolomics, UPLC-Q-TOF-MS.

\section{INTRODUCTION}

Alzheimer's disease (AD) is a common neurodegenerative disease of the elderly, mainly in the old age [1]. Memory decline, cognitive dysfunction, behavioral abnormalities and social barriers are the main clinical manifestations of AD. The disease is a heterogeneous disease, which can be caused by various factors, including biological and psychosocial factors. The etiology and pathogenesis of AD have not been elucidated, and the characteristic pathological changes are neurogenic fibrous tangles formed by hyperphosphorylation of extracellular matrix and tau protein formed by amyloidosis of beta amyloid, as well as neuron loss and glial proliferation [2]. Although cholinesterase inhibitors have been used as the main means to treat $\mathrm{AD}$, there are a series of problems including slow onset, relapse and many adverse reactions. At present, the development of anti-AD drugs has shifted to traditional Chinese medicine (TCM). TCM is composed of two or more than two flavors. It is the main component of Chinese medicine compatibility and the main means of modern Chinese medicine clinical treatment Ginsenoside and tenuigenin are the major effective compounds found in ginseng and polygala tenuifolia, respectively. Ginseng and polygala tenuifolia are frequently used in the clinical treatment of $\mathrm{AD}$, and their representative prescriptions, such as Kai-Xin San, Ding-Zhi-Xiao-Wan have shown good anti-AD effect in animal experiments and clinical study $[3,4]$.

Metabonomics is a newly developed discipline after genomics and proteomics [5]. It is an important part of systems biology [6]. The systematic characteristics of metabonomics conform to the overall characteristics of TCM [7]. High resolution mass spectrometry (MS) is widely used in metabonomics because it can obtain more specific and accurate quality in metabolite identification [8]. In recent years, with the development of mass spectrometry and the development of the interface of chromatography-mass spectrometry, liquid and mass spectrometry has played an important role in drug analysis [9]. It is an indispensable part of modern drug research, and it is also the most powerful tool for the research of Chinese medicine compound [10]. Liquid chromatographymass spectrometry (LC-MS) avoids the complex pretreatment of the TCM [11]. In this study, ultra-performance liquid chromatography coupled with quadrupole time-of-flight high definition mass spectrometry (UPLC-QTOF-MS) method was established to obtain endogenous metabolites in urine of $\mathrm{AD}$ rats. Pattern recognition method was used to study the changes of metabolite pathway to further understand how ginsenosides and tenuigenins compatibility modifies symptom AD.

\section{MATERIALS AND METHODS}

\subsection{Materials}

Ginsenosides and tenuigenins were purchased from College of Chemistry, Jilin University (Changchun, Jilin, China). Wistar rats, male, weighing 180$220 \mathrm{~g}$, were purchased from the animal experimental center of Bethune Medical College, Jilin University. Aluminium trichloride $\left(\mathrm{AlCl}_{3}\right)$ (Maoming Chemical Reagent Factory, Tianjin), D-galactose (D-gal) (ICA biotech, Shanghai), Leucine enkephalin and sodium for mate were obtained from Waters (Milford, USA). Acetonitrile and formic acid (Fisher Scientific, Lough borough, UK) were HPLC-grade. Ultrapure water was produced by a Milli-Q plus (Milford, MA, USA) water purification system.

\subsection{AD model construction and treatment}

A total of 30 Wistar rats were bred for 1 weeks. Then, they were randomly divided into 3 groups: normal group (NG), model group (MG) and treatment group (TG). Each group comprised 10 rats. MG and the TG rats were intragastrically administered with $\mathrm{AlCl}_{3} \quad(200 \mathrm{mg} / \mathrm{kg})$ and intraperitoneally administered with D-gal $(60 \mathrm{mg} / \mathrm{kg})$ every day for 8 
weeks to establish AD model. 4 weeks after modeling, the rats in the TG were given compatibility of ginsenosides and tenuigenins (ginsenosides $200 \mathrm{mg}$ and tenuigenins $400 \mathrm{mg}$ ) per kg per day for 4 weeks.

\subsection{Urine sample preparation}

Samples of $24 \mathrm{~h}$ urine were collected on the 28th day. Before UPLC-MS analysis, the urine was thawed at room temperature. After12000 rpm centrifugation for $10 \mathrm{~min}$, the urine was diluted 10 times with purified water, and then filtered through $0.22 \mathrm{M}$ filter membrane.

\subsection{UPLC-MS conditions}

The Waters Acquity UPLC liquid chromatography system, Q-TOF SYNAPT G2 HDMS mass spectrometer and Waters ACQUITY UPLC BEH C18 chromatographic column $(2.1 \mathrm{~mm} \times 50 \mathrm{~mm}, 1.7 \mu \mathrm{m})$ were used for the analysis of liquid chromatography mass spectrometry. The column temperature is $35^{\circ} \mathrm{C}$, the sampling amount is $5 \mathrm{uL}$, the flow phase A phase is $0.1 \%$ formic acid $(\mathrm{v} / \mathrm{v})$, the $\mathrm{B}$ phase is acetonitrile, the flow rate is 0.4 $\mathrm{mL} / \mathrm{min}$, the gradient elution condition is $5-20 \%$ B $0-3 \mathrm{~min}, 20-40 \%$ B 3-6 min, $40-100 \%$ 6-8min, $100 \%$ B 8-10 min, $100-5 \%$ B $10-10.1$ min, $5 \%$ B 10.1-14min.

Mass spectrometry using ESI ionization source, the mass scanning range is $\mathrm{m} / \mathrm{z} 100-1000 \mathrm{Da}$. The temperature of ionization source is 120 degrees, the temperature of desolvent gas is 350 , the gas of taper hole and desolvent are all nitrogen, and the flow rate is $50 \mathrm{~L} / \mathrm{h}$ and $700 \mathrm{~L} / \mathrm{h}$ respectively. Under the positive ion mode, the capillary voltage is $3 \mathrm{kV}$, the cone hole voltage is $30 \mathrm{~V}$, the extracting cone voltage is $5 \mathrm{~V}$, the capillary voltage is $2 \mathrm{kV}$ under the negative ion mode, the cone whole voltage is $30 \mathrm{~V}$, and the extraction cone whole voltage is $5 \mathrm{~V}$. Sodium formate was used to establish the quality axis standard curve and leucine enkephalin was corrected in real time. The MSE method is used for MS/MS analysis. Argon is used as a collision gas, with a low collision energy of $5 \mathrm{eV}$ and a high collision energy of $25-35 \mathrm{eV}$.

\subsection{Data processing and statistical analysis}

Raw data is first processed by MarkerLynx application manager and MassLynxV4.1 for compound detection. Then the exact mass, retention time and the intensities as a data matrix were imported into EZinfo 2.0, a multivariate statistical analysis software. Biochemical databases are used to identify potential markers, such as HMDB (http://www.hmdb.ca/), MetaboAnalyst (http://www.metaboanalyst.ca/).

\section{RESULTS AND DISCUSSION}

\subsection{Urine metabonomic profiles analysis}

Both positive and negative ion modes were used to acquire urinary metabonomic profiles. Figure 1 shows urinary representative based peak intensity (BPI) chromatograms of three groups in the positive and negative ion modes. The biggest feature of metabonomics data is that the number of variables is far greater than the number of observations, and there is a high degree of correlation between variables. Although differences in the BPI chromatograms of the 3 groups were observed, multivariate analysis method such as PCA and OPLS-DA could represent the differences more clearly. PCA is considered as the starting point and provides an overview of the information hidden in multivariate data. Each point of PCA score represented an individual sample. Through PCA analysis (Figure 2A-B), the 3 groups could be clearly separated, indicating that group difference was more remarkable than individual difference. OPLS-DA can enhance the separation between groups of observations and improve the interpretation of models. As shown in OPLS-DA plots (Figure 2C-D), the MG and TG were definitely divided into 2 classes. S-plots are usually used to identify the features contributing to the group discrimination. Each point in the S-plots represents an endogenous substance. As shown in Figure 2E-F, the points located at the ends of the S-plot contributed to the differentiation between two groups. The R2Y and Q2Y were 0.98 and 0.92 in positive ion mode, respectively; whereas the R2Y and Q2Y were 0.96 and 0.94 in negative ion mode, respectively. These findings indicated that the models were valid and reliable.
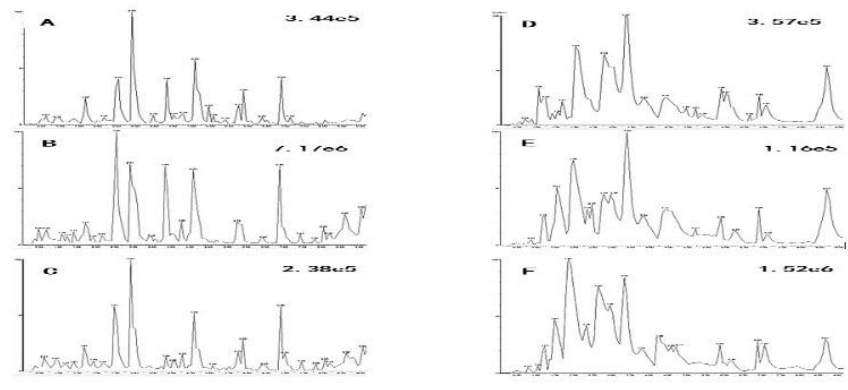

Figure 1: Urinary base peak chromatograms of $N G(A), M G$ (B) and TG (C) in positive mode and NG (D), MG (E) and TG (F) in negative mode.
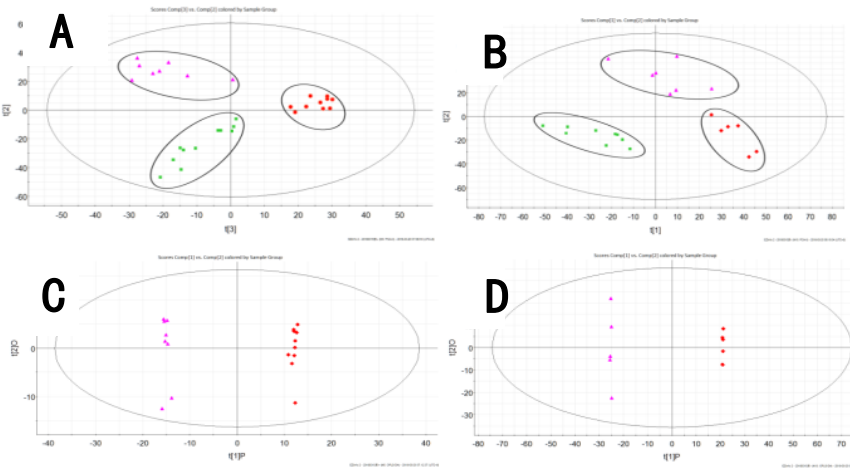

D
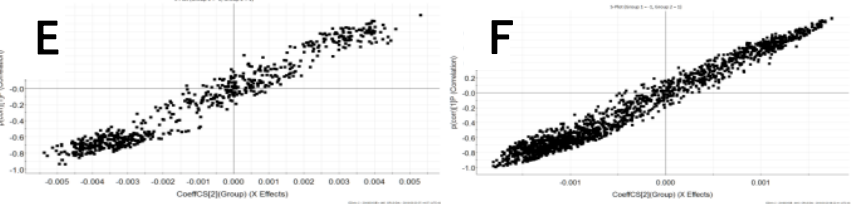

Figure 2: PCA score plots of rat urine metabolic profiles of NG, MG and $\mathrm{TG}$ in positive ion mode $(\mathrm{A})$ and negative ion mode(B) $\left(\Delta \mathrm{TG} \square_{\mathrm{NG}}\right.$ $M G)$. OPLS-DA score plots of urine metabolic profiling positive ion mode (C) and negative ion mode(D) ( $\Delta_{\mathrm{TG}} \Delta_{\mathrm{MG}}$ ). S-plots of urine metabolic profiling positive ion mode $(\mathrm{E})$ and negative ion mode $(\mathrm{F})$.

Table 1: Identification results of potential biomarkers

\begin{tabular}{|c|c|c|c|c|c|c|}
\hline Mode & $\begin{array}{l}\text { RT } \\
(\mathrm{min})\end{array}$ & $\begin{array}{l}\text { Measured } \\
\text { Mass }\end{array}$ & VIP & $\begin{array}{l}\text { Error } \\
(\mathrm{ppm})\end{array}$ & Formula & Identitification \\
\hline \multirow[t]{3}{*}{ ESI+ } & 3.89 & 349.1535 & 1.14 & -1.0 & $\mathrm{C}_{17} \mathrm{H}_{20} \mathrm{FN}_{3} \mathrm{O}_{4}$ & Pefloxacin $\mathrm{N}$-oxide \\
\hline & 1.54 & 163.0702 & 1.12 & -0.9 & $\mathrm{C}_{17} \mathrm{H}_{20} \mathrm{FN}_{3} \mathrm{O}_{4}$ & 3-Methyldioxyindole \\
\hline & 0.74 & 298.0708 & 1.06 & -1.0 & $\mathrm{C}_{12} \mathrm{H}_{14} \mathrm{~N}_{2} \mathrm{O}_{5} \mathrm{~S}$ & N-Acetylserotonin sulfate \\
\hline \multirow[t]{7}{*}{ ESI- } & 0.45 & 254.0498 & 1.38 & -1.0 & $\mathrm{C}_{7} \mathrm{H}_{14} \mathrm{~N}_{2} \mathrm{O}_{6} \mathrm{~S}$ & 5-L-Glutamyl-taurine \\
\hline & 8.82 & 594.3309 & 1.33 & -0.1 & $\mathrm{C}_{7} \mathrm{H}_{14} \mathrm{~N}_{2} \mathrm{O}_{6} \mathrm{~S}$ & L-Urobilin \\
\hline & 10.6 & 334.2098 & 1.31 & 1.0 & $\mathrm{C}_{20} \mathrm{H}_{30} \mathrm{O}_{4}$ & $\begin{array}{l}\text { 7'-Carboxy-gamma- } \\
\text { chromanol } \\
\text { (E)-2-octenal }\end{array}$ \\
\hline & 4.12 & 126.0974 & 1.20 & 1.0 & $\mathrm{C}_{8} \mathrm{H}_{14} \mathrm{O}$ & \\
\hline & 0.45 & 242.0126 & 1.10 & 1.0 & $\mathrm{C}_{6} \mathrm{H}_{11} \mathrm{O}_{8} \mathrm{P}$ & Inositol cyclic phosphate \\
\hline & 9.1 & 206.0524 & 1.16 & 1.0 & $\mathrm{C}_{11} \mathrm{H}_{10} \mathrm{O}_{4}$ & $\begin{array}{l}\text { 6-(hydroxymethyl)-7- } \\
\text { methoxy-2H-chromen-2- } \\
\text { one } \\
\text { Inositol phosphate }\end{array}$ \\
\hline & 0.44 & 260.0209 & 1.31 & 0.9 & $\mathrm{C}_{6} \mathrm{H}_{13} \mathrm{O}_{9} \mathrm{P}$ & \\
\hline
\end{tabular}

\subsection{Identification of potential biomarkers}

The threshold for selecting potential biomarkers was set at Variable importance in the projection $>1.0$ and $\mathrm{p}<0.05$. By comparing the discrimination of three groups using the OPLS-DA model, ten endogenous 
metabolites in urine were considered to be potential biomarkers correlated with how compatibility of ginsenosides and tenuigenins influenced $\mathrm{AD}$ rats. The possible molecular formula of the potential biomarker was calculated by high-accuracy quasi-molecular ion within a mass error of $10 \mathrm{ppm}$, and fractional isotope abundance was detected by Q-TOF-MS. Structure information was obtained by searching freely accessible databases, such as HMDB, METLIN, Massbank, and KEGG. MS/MS fragmentation patterns also provided necessary information for the structure of biomarkers. Some of these biomarkers were confirmed with available reference standards by matching their retention time and accurate mass measurement. Other biomarkers were presumed on based on accurate elemental compositions and MS/MS behavior. The information on potential biomarkers is summarized in Table 1.

\subsection{Metabonomic pathway analysis}

The metabonomic tool was also used to perform pathway analysis. By importing the potential biomarkers, an integral pathway analysis can be carried out. As shown in Figure 3 and Table 2, the main metabonomic pathways affected by the compatibility of ginsenosides and tenuigenins administration in $\mathrm{AD}$ rats were taurine and hypotaurine metabolim, inositol phosphate metabolism and Tryptophan metabolism.

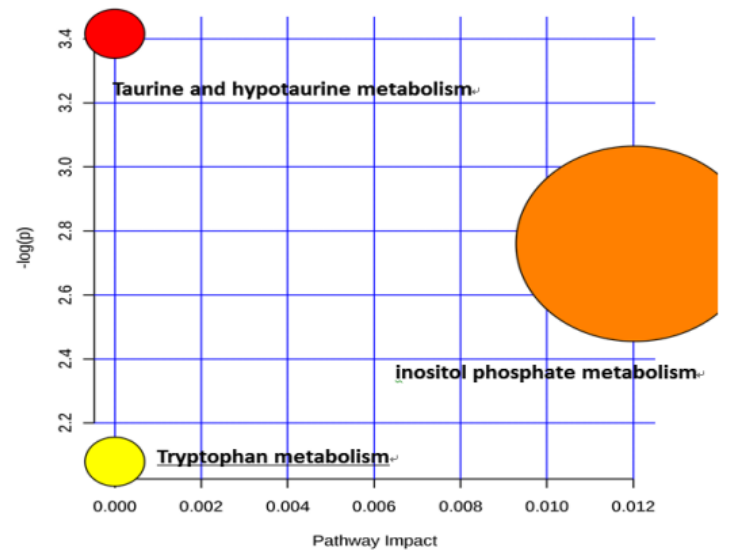

Figure 3: Overview of pathway analysis

Table 2: The main pathway affects by and ginsenosides and tenuigenins on AD

\begin{tabular}{|cccccc|}
\hline Pathway & Total & Expected & Hits & Pvalue & Impact \\
\hline Taurine and hypotaurine metabolim & 20 & 0.041 & 1 & 0.041 & 0.003 \\
Inositol phosphate metabolism & 39 & 0.081 & 1 & 0.078 & 0.012 \\
Tryptophan metabolism & 79 & 0.164 & 1 & 0.153 & 0.002 \\
\hline
\end{tabular}

\subsection{Biological meaning}

The interpretation of the biomarker was shown in Figure 4. Among these metabolites, 3-methyldioxyindole are downstream metabolites of tryptophan pathway [12]. Tryptophan, an essential amino acid, plays a fundamental role in physiology and biochemistry [13]. It is either incorporated into proteins or broken down for intermediates of energy metabolism. Tryptophan metabolism through kynurenine pathway was considered as one of many mechanisms that are involved in how immune system continuously modulates the balance between responsiveness to pathogens and tolerance of non-harmful antigens [14]. Tryptophan metabolism impacts the immune system mainly via the kynurenine pathway, in which indoleamine-2,3-dioxygenase plays a pivotal role as a rate-limiting. Kynurenic acid could reduce $\mathrm{N}$-methyl-D-aspartate receptor activity and disturb brain functions as a neuro-protective molecule [15]. Studies have shown that 3-Methyl-dioxyindole has an effect on the tryptophan metabolism pathway in Alzheimer's disease rats.

5-L-Glutamyl-taurine is an intermediate in taurine and hypotaurine metabolism. Taurine, hypotaurine and their metabolic precursors act as antioxidants in vivo [16]. Taurine, synthesised via cysteine and vitamin $B_{6}$, is an essential amino acid and acts as the most important Inhibitory neurotransmitter in the brain. The biological functions of taurine include anti-oxidation, $\mathrm{Ca}^{2+}$ transport regulation, and anti-inflammation. taurine chloramine could suppress the secretion of TNF- $\alpha$ in vivo [17]. The taurine and hypotaurine metabolism pathway was themajor metabolic pathway in the ginsenoside and tenuigenin for treating AD rats.

Inositol phosphate come from the enzymatic hydrolysis of phosphatidylinositol in vivo. Many kinds of phosphoinositide play an important role in information substance [18].The most important is three phosphoinositide, which is the hydrolysate of triphosphoinositide phosphatide [19]. Its main role is to induce the release of $\mathrm{Ca}^{2+}$ from the intracellular reservoir and instantly increase the concentration of $\mathrm{Ca}^{2+}$ in the cytosol. Because this process is based on the fact that $\mathrm{Ca}^{2+}$ is a widespread intracellular messenger substance, this action can play an important role in regulating cell responses.

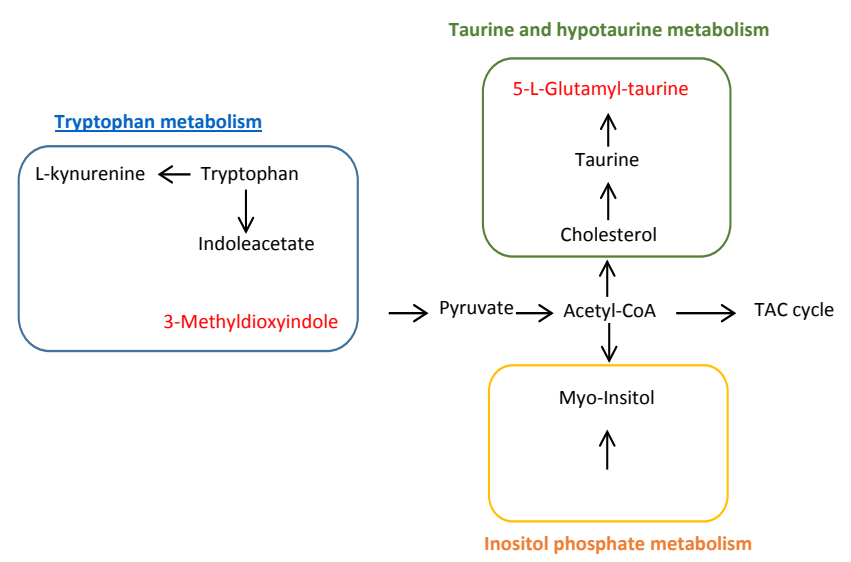

Figure 4: Correlation networks of potential biomarkers involved in the response to the therapeutic effects ofginsenoside and tenuigenin in $\mathrm{AD}$ rats. The metabolites written in red are identified as potential biomarkers.

\section{CONCLUSION}

The present study demonstrated that compatibility of ginsenosides and tenuigenins had an anti-AD effect on $\mathrm{AD}$ rats. The mechanism of anti-AD effect was partially relevant with regulating ten potential biomarkers levels. These biomarkers were belong to tryptophan metabolism, taurine and hypotaurine metabolism and inositol phosphate metabolism.

\section{REFERENCES}

[1] Stites, S.D., Milne, R., Karlawish, J. 2018. Advances in Alzheimer's imaging are changing the experience of Alzheimer's disease. Alzheimer's \& Dementia: Diagnosis, Assessment \& Disease Monitoring, 10, 285-300.

[2] Calderon-Garciduenas, A.L., Duyckaerts, C. 2017. Alzheimer disease. Clinical neurology and neurosurgery, 145, 325-337.

[3] Meng, S., Lin, Z., Wang, Y., Wang, Z., Li, P., Zheng, Y. 2018. Psoriasis therapy by Chinese medicine and modern agents, $13,16$. 
[4] Dong, H., Wu, S., Hu, N., Xing, G. 2018. Efficacy of tenuigenin and betaasarone as augmentations for memantine in the treatment of Alzheimer's disease. Journal of pharmaceutical and biomedical analysis 29 (3), 203207.

[5] Jiang, W., Gao, L., Li, P., Kan, H., Qu, J., Men, L., Liu, Z., Liu, Z. 2017. Metabonomics study of the therapeutic mechanism of fenugreek galactomannan on diabetic hyperglycemia in rats, by ultra-performance liquid chromatography coupled with quadrupole time-of-flight mass spectrometry. Journal of Chromatography B, 1044-1045, 8-16.

[6] Gika, H.G., Theodoridis, G.A., Plumb, R.S., Wilson, I.D. 2014. Current practice of liquid chromatography-mass spectrometry in metabolomics and metabonomics. Journal of pharmaceutical and biomedical analysis, 87 , $12-25$.

[7] Chen, Z., Cao, Y., He, S., Qiao, Y. 2018. Development of models for classification of action between heat-clearing herbs and blood-activating stasis-resolving herbs based on theory of traditional Chinese medicine. Chinese Medical Journal, 13, 12.

[8] Matysik, S., Liebisch, G. 2017. Quantification of steroid hormones in human serum by liquid chromatography-high resolution tandem mass spectrometry. Journal of chromatography A, 1526, 112-118.

[9] Bylinski, H., Gebicki, J. 2017. Direct Analysis of Samples of Various Origin and Composition Using Specific Types of Mass Spectrometry, 47 (4), 340-358.

[10] Ojanpera, I., Kolmonen, M., Pelander, A. 2012. Current use of highresolution mass spectrometry in drug screening relevant to clinical and forensic toxicology and doping control. Analytical and bioanalytical chemistry, 403 (5), 1203-1220.

[11] Li, X., Li, H., Ma, W., Guo, Z., Li, X., Li, X., Zhang, Q. 2018. Determination of patulin in apple juice by single-drop liquid-liquid-liquid microextraction coupled with liquid chromatography-mass spectrometry. Food chemistry, 257, 1-6.

[12] Hunt, N.H., Too, L.K., Khaw, L.T., Guo, J., Hee, L., Mitchell, A.J., Grau, G.E., Ball, H.J. 2017. The kynurenine pathway and parasitic infections that affect CNS function. Neuropharmacology, 112, Part B, 389-398.

[13] Davis, I.S. 2015. 40. Medicine \& Science in Sports \& Exercise, 47(5S).

[14] Blei, F., Baldeweg, F., Fricke, J., Hoffmeister, D. 2018. Biocatalytic production of psilocybin and derivatives in tryptophan synthaseenhanced reactions. Chemistry.

[15] Fernandes, J., Mudgal, J.2018. N-acetyl-L-tryptophan, a substance-P receptor antagonist attenuates aluminum-induced spatial memory deficit in rats. 28 (5), 328-334.

[16] Alfano, M., Poli, G. 2005. Role of cytokines and chemokines in the regulation of innate immunity and HIV infection. Molecular Immunology, $42(2), 161-182$.

[17] Imae, M., Asano, T., Murakami, S. 2014. Potential role of taurine in the prevention of diabetes and metabolic syndrome. Amino Acids, 46 (1), 81 88.

[18] Sjoberg, P.J.R., Thelin, P., Rydin, E. 2016. Separation of inositol phosphate isomers in environmental samples by ion-exchange chromatography coupled with electrospray ionization tandem mass spectrometry. Talanta, 161, 392-397.

[19] Hanke, D.E., Parmar, P.N., Caddick, S.E., Green, P., Brearley, C.A. 2012. Synthesis of inositol phosphate ligands of plant hormone-receptor complexes: pathways of inositol hexakisphosphate turnover. biochemical journal, 444 (3), 601-609. 